\title{
Dysregulated gliotoxin biosynthesis attenuates the production of unrelated biosynthetic gene cluster-encoded metabolites in Aspergillus fumigatus
}

\author{
Sean Doyle ${ }^{\mathrm{a}}$, Gary W. Jones ${ }^{\mathrm{a}, \mathrm{b}}$, Stephen K. Dolan ${ }^{\mathrm{a}, \mathrm{c} \text {, * }}$ \\ a Department of Biology, Maynooth University, Maynooth, Co. Kildare, Ireland \\ b Centre for Biomedical Science Research, Leeds Beckett University, Leeds LS1 3HE, UK \\ ${ }^{\mathrm{c}}$ Department of Biochemistry, University of Cambridge, Cambridge CB2 1QW, UK
}

\section{A R T I C L E I N F O}

\section{Article history:}

Received 21 July 2017

Received in revised form

20 November 2017

Accepted 10 December 2017

Available online 18 December 2017

Corresponding Editor: Gustavo Henrique Goldman

\section{Keywords:}

BGC

Fungal proteomics

LC-MS

Metabolomics

Methyltransferase

NRPS

\begin{abstract}
A B S T R A C T
Gliotoxin is an epipolythiodioxopiperazine (ETP) class toxin, contains a disulfide bridge that mediates its toxic effects via redox cycling and is produced by the opportunistic fungal pathogen Aspergillus fumigatus. The gliotoxin bis-thiomethyltransferase, GtmA, attenuates gliotoxin biosynthesis in A. fumigatus by conversion of dithiol gliotoxin to bis-thiomethylgliotoxin (BmGT). Here we show that disruption of dithiol gliotoxin bis-thiomethylation functionality in A. fumigatus results in significant remodelling of the A. fumigatus secondary metabolome upon extended culture. RP-HPLC and LC-MS/MS analysis revealed the reduced production of a plethora of unrelated biosynthetic gene cluster-encoded metabolites, including pseurotin $\mathrm{A}$, fumagillin, fumitremorgin $\mathrm{C}$ and tryprostatin $\mathrm{B}$, occurs in A. fumigatus $\triangle g t m A$ upon extended incubation. Parallel quantitative proteomic analysis of $A$. fumigatus wild-type and $\triangle g t m A$ during extended culture revealed cognate abundance alteration of proteins encoded by relevant biosynthetic gene clusters, allied to multiple alterations in hypoxia-related proteins. The data presented herein reveal a previously concealed functionality of GtmA in facilitating the biosynthesis of other BGC-encoded metabolites produced by $A$. fumigatus.
\end{abstract}

(C) 2017 British Mycological Society. Published by Elsevier Ltd. All rights reserved.

\section{Introduction}

Ascomycetes constitute the largest phylum of the fungal kingdom and produce an incredible array of natural products. Although many of these compounds are known as medicinal therapeutics or industrial chemicals, several natural products are potent toxins which pose substantial threats to human food supplies and health (Schueffler and Anke, 2014). The opportunistic fungus Aspergillus fumigatus produces a wealth of these potent natural product toxins which are encoded by multigene biosynthetic gene clusters (BGCs) (Bignell et al., 2016; Lind et al., 2017).

Gliotoxin has been shown to be a virulence attribute in invasive aspergillosis due to its cytotoxic, genotoxic and apoptosisstimulating properties. It is produced through a sequential series of enzymatic steps, which are predominantly encoded by the gli BGC (Dolan et al., 2015). Gliotoxin biosynthesis is also influenced by

\footnotetext{
* Corresponding author. Department of Biochemistry, University of Cambridge, Cambridge CB2 1QW, UK.
}

E-mail address: skd41@cam.ac.uk (S.K. Dolan).
GtmA (TmtA in Scharf et al. (2014)), a thiol-directed methyltransferase encoded outside the gli BGC, which specifically bisthiomethylates both thiols of dithiol gliotoxin to form bis-thiomethylgliotoxin (BmGT), resulting in the attenuation of gliotoxin formation (Dolan et al., 2014). Lines between defined BGCs have been blurred by elegant work describing the existence of intertwined biosynthetic gene clusters which are involved in the formation of more than one chemical product (Wiemann et al., 2013). For example, A. fumigatus encodes a "supercluster" in the subtelomeric region of chromosome 8 , in which the genes responsible for the production of two natural products, pseurotin and fumagillin, are physically intertwined (Wiemann et al., 2013). Like gliotoxin, these other natural products also have potent toxicities. For example, pseurotin $\mathrm{A}$ is an immunosuppressive spirocyclic that has been shown to have interesting biological activities including the ability to induce the cellular differentiation of PC12 neuronal cells (Komagata et al., 1996), monoamine oxidase inhibitory activity (Maebayashi et al., 1985) and chitin synthase inhibitory activity (Wenke et al., 1993), highlighting many potential applications of 
this metabolite. Fumagillin has antibiotic and antifungal activity. It was also found to exhibit anti-cancer properties and antiangiogenic activity as a inhibitor of the human type 2 methionine aminopeptidase (MetAP2) (Sin et al., 1997; Hou et al., 2009).

Despite the fact that BGCs often possess a pathway-specific transcription factor, the production of several otherwise unconnected natural products has been shown to be regulated by global regulators of secondary metabolism such as LaeA. This functionally enigmatic regulator has been shown to regulate the production of several A. fumigatus secondary metabolites (SMs) including gliotoxin, fumagillin, pseurotin A and helvolic acid (Perrin et al., 2007). LaeA has also been shown to counteract the establishment of heterochromatin marks, thus activating SM production, inferring that LaeA regulates BGC-encoded metabolism by modifying chromatin structure (Nützmann et al., 2011). This suggests that fungal secondary metabolism is controlled by a rigorous hierarchy of regulatory mechanisms.

Understanding the diversity of regulatory strategies controlling the expression of these pathways is therefore critical if their biosynthetic potential is to be explored for new drug leads. No single medium under standardized growth conditions can secure expression of the full potential for producing the secondary metabolome of a fungal culture. However, there are many strategies to enable fungal cultures to produce even more BGC-encoded metabolites; for instance by modifying the media constituents, adding other microorganisms, using light or using longer incubation periods, alternate temperatures or deploying low or high $\mathrm{pH}$ (Ochi and Hosaka, 2013).

The filamentous fungus Aspergillus terreus produces the ETP acetylaranotin (Guo et al., 2013). Similar to gliotoxin, this metabolite and its derivatives have been shown to display an array of interesting bioactivities including the induction of apoptosis in cancer cell lines and antifungal activity (Guo et al., 2013; Choi et al., 2011; Suzuki et al., 2000; Li et al., 2016). Three novel bis(methylthio) dioxopiperazine derivatives of the epipolythiodioxopiperazine (ETP) emestrin were produced by Podospora australis following static incubation of cultures at $23^{\circ} \mathrm{C}$ for $14 \mathrm{~d}$ (Li et al., 2016). Like P. australis, A. fumigatus produces many thiomethylated forms of gliotoxin upon incubation for two weeks at $25^{\circ} \mathrm{C}$ in the dark (Forseth et al., 2011). Extending the incubation duration of $A$. terreus cultures from $6 \mathrm{~d}$ to $42 \mathrm{~d}$ resulted in the production of four thiomethylated forms of acetylaranotin, which were undetectable at earlier incubation time points. One of these bioactive SMs (bisdethiobis(methylsulfanyl) apoaranotin) exhibited growth inhibitory properties against $\mathrm{Myco}$ bacterium tuberculosis H37Ra with an MIC value of $1.56 \mu \mathrm{g} / \mathrm{ml}$, thus highlighting the potential of extended incubation to develop exciting natural product derivatives (Haritakun et al., 2012). As the generation of these thiomethylated forms of acetylaranotin was likely dependent on an A. terreus GtmA homolog, this research prompted us to explore the effect of extended culture incubation on A. fumigatus wild-type and $\Delta g t m A$. Moreover, despite significant work describing the production and isolation of these ETP derivatives (Dolan et al., 2014; Scharf et al., 2014; Liang et al., 2014), the downstream effects of removing this ETP bis-thiomethylation functionality and concomitant perpetuation of gliotoxin biosynthesis, have not been explored to date in extended cultures.

\section{Materials and methods}

\subsection{RP-HPLC and LC-MS detection of natural products from} A. fumigatus culture supernatants

A. fumigatus wild-type, $\triangle g t m A$ and $g t m A^{\mathrm{c}}$ strains (Dolan et al., 2014) were grown $\left(10^{8}\right.$ conidia $\left./ \mathrm{ml}\right)$ in quadruplicate (Czapek-Dox broth, $200 \mathrm{rpm}, 3 \mathrm{~d}$; then static, $25 \mathrm{~d} 37^{\circ} \mathrm{C}$ ). Culture supernatants and ethyl acetate organic extracts $(100 \mu \mathrm{l})$ were analysed by RPHPLC with UV detection (Agilent 1200 system), using a C18 RPHPLC column (Agilent Zorbax Eclipse XDB-C18 Semi-Preparative; $5 \mu \mathrm{m}$ particle size; $4.6 \times 250 \mathrm{~mm}$ ) at a flow rate of $2 \mathrm{ml} / \mathrm{min}$ (Fig 1 ). A mobile phase of water and acetonitrile with $0.1 \%(\mathrm{v} / \mathrm{v})$ trifluoroacetic acid, was used under various gradient conditions. For LC-MS analysis, organic extracts were diluted $1 / 10$ in $0.1 \%(\mathrm{v} / \mathrm{v})$ formic acid and spin filtered prior to LC-MS analysis (Agilent Ion Trap 6340). Gliotoxin (purity: 98 \%) and BmGT (purity: $99 \%$ ) standards were obtained from Sigma-Aldrich and Enzo Life Sciences, respectively. Fumagillin, pseurotin A, tryprostatin B and fumitremorgin $C$ were identified based on published $m / z$ ratios, retention times and fragmentation patterns as described previously (O'Keeffe et al., 2014). All data were analysed using built-in GraphPad prism version 5.01 functions, as specified. The level of significance was set at $p<0.05\left(^{*}\right), p<0.001\left(^{* *}\right)$, and $p<0.0001$ $\left({ }^{* * *}\right)$, unless otherwise stated.

\subsection{Comparative quantitative proteomic analysis of A. fumigatus wild-type and mutant strains}

As shown in Fig 1, mycelia were then harvested and snap frozen in liquid $\mathrm{N}_{2}$. No significant differences in biomass were noted for these strains (Supplementary Figs 1 and 2). Mycelial lysates were prepared in lysis buffer (100 mM Tris- $\mathrm{HCl}, 50 \mathrm{mM} \mathrm{NaCl}, 20 \mathrm{mM}$ EDTA, $10 \%$ (v/v) glycerol, 1 mM PMSF, $1 \mu \mathrm{g} / \mathrm{ml}$ pepstatin A, pH 7.5) with grinding, sonication and clarified using centrifugation. The resultant protein lysates were precipitated using trichloroacetic acid/acetone and resuspended in $100 \mathrm{mM}$ Tris- $\mathrm{HCl}, 6 \mathrm{M}$ urea, $2 \mathrm{M}$ thiourea, $\mathrm{pH}$ 8.0. After dithiothreitol reduction and iodoacetamidemediated alkylation, sequencing grade trypsin combined with ProteaseMax surfactant was added. All peptide mixtures were analysed via a Thermo Fisher Q-Exactive mass spectrometer coupled to a Dionex RSLCnano. LC gradients ran from 4 to 35 \% B over $2 \mathrm{~h}$, and data was collected using a Top15 method for MS/MS scans. Comparative proteome abundance and data analysis was
A. fumigatus Shaking Culture
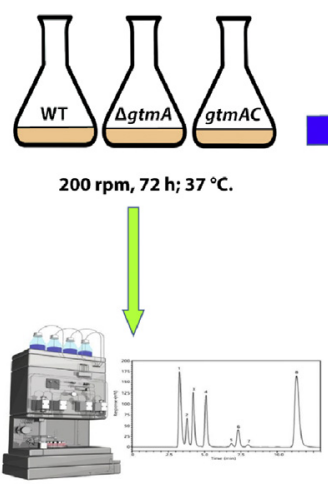

RP-HPLC Analysis
A. fumigatus Static Culture
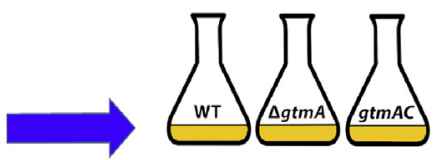

Static, $25 \mathrm{~d} ; 37^{\circ} \mathrm{C}$.

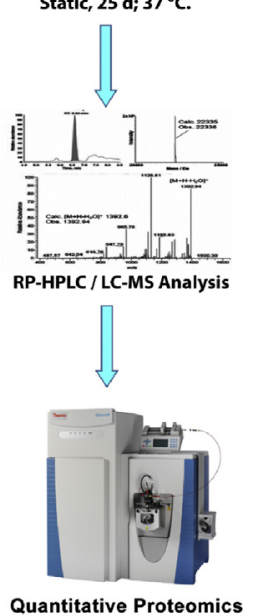

Fig 1. Flow diagram describing the experimental setup. Wild-type, $\Delta \operatorname{gtm} A$ and $\operatorname{gtm} A^{\mathrm{C}}$ were grown for $3 \mathrm{~d}$ shaking at $37^{\circ} \mathrm{C}$. Cultures where then incubated at $37^{\circ} \mathrm{C}$, static for a further $25 \mathrm{~d}$. Culture supernatants were analysed by RP-HPLC at $3 \mathrm{~d}$, Samples were analysed by RP-HPLC, LC-MS/MS and quantitative proteomics at $28 \mathrm{~d}$. 
A.

B.

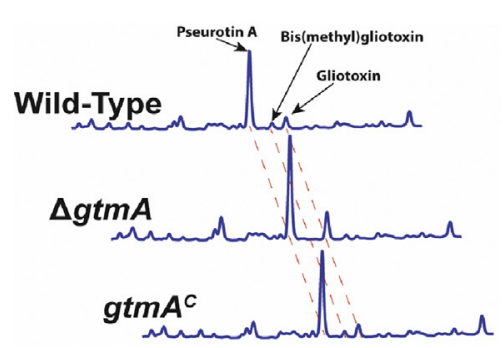

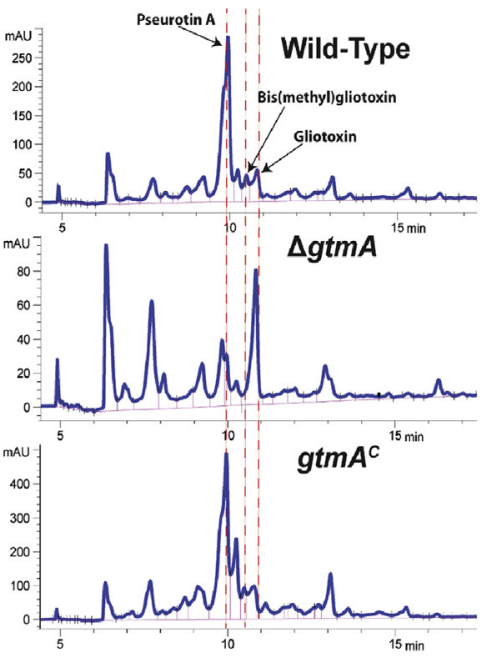

C.

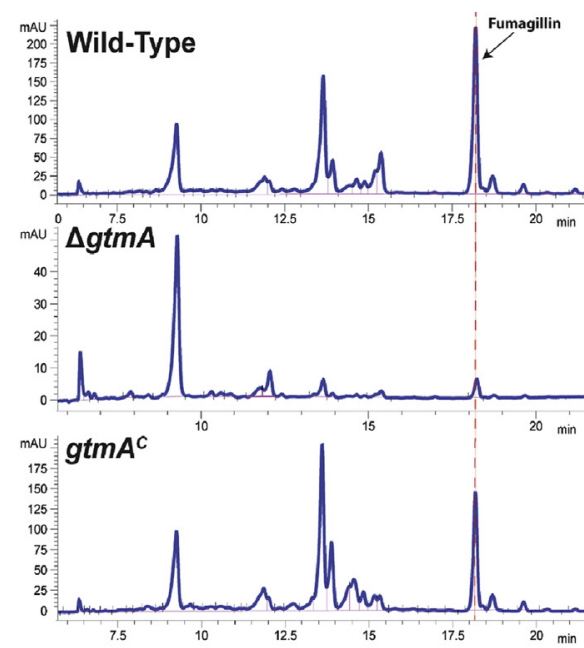

D.
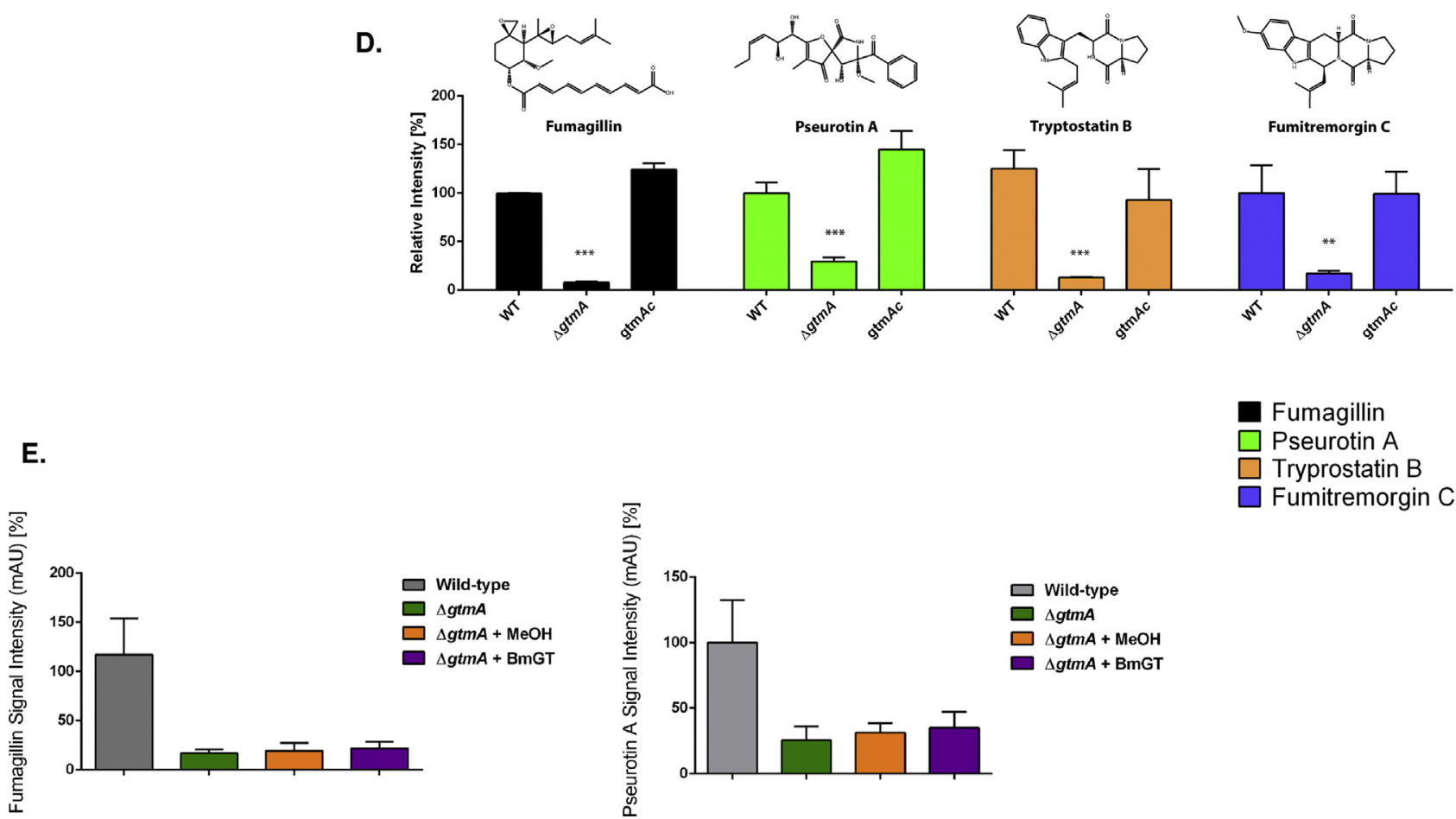

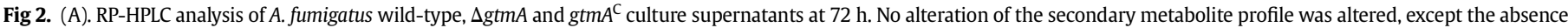

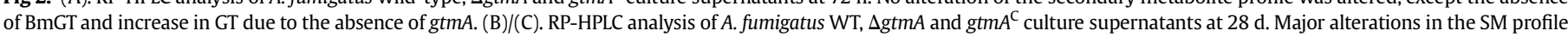

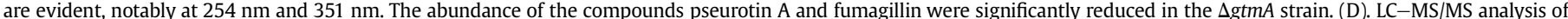

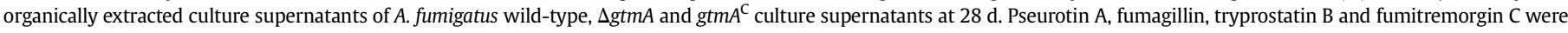

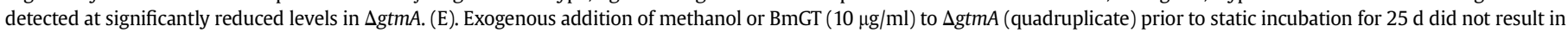
the restoration of pseurotin A or fumagillin production levels to that of the wild-type strain. Bars represent pseurotin A or fumagillin RP-HPLC intensity (mAU) at 254 nm.

performed using MaxQuant software (Version 1.3.0.5), with Andromeda used for database searching and Perseus used to organise the data (Version 1.4.1.3) (Cox and Mann, 2008).

\section{Results and discussion}

3.1. Late-stage culture metabolomics reveals that dysregulated gliotoxin biosynthesis due to gtmA absence influences the biosynthesis of other secondary metabolites

Comparative RP-HPLC analysis of culture supernatants at $3 \mathrm{~d}$ showed that the overall SM profile was not altered significantly by the deletion of gtmA (Fig 2A), except that gliotoxin production was significantly increased and bis-thiomethylgliotoxin (BmGT) production was abolished as described previously (Dolan et al., 2014). However, when the culture incubation time was increased by $25 \mathrm{~d}$, a decrease in several compounds was specifically detected in $A$. fumigatus $\Delta$ gtmA by RP-HPLC DAD (at $254 \mathrm{~nm}$ and $351 \mathrm{~nm}$ ) (Fig 2B and $\mathrm{C}$ ). The production of these compounds was restored in the complemented strain $\left(g t m A^{\mathrm{c}}\right)$. These compounds were fractioncollected, analysed by LC-MS and identities were elucidated based on known $m / z$ values and fragmentation patterns. Pseurotin A $(p=0.0001)$ and fumagillin $(p=0.0005)$ were produced at significantly decreased concentrations in A. fumigatus $\Delta g t m A$ (Fig 
2D). Furthermore, LC-MS/MS analysis of ethyl-acetate extracted culture supernatants also uncovered that the detected concentration of tryprostatin B $(p=0.0005)$ and fumitremorgin $\mathrm{C}$ $(p=0.0074)$ were significantly lower in the $\Delta g t m A$ strain (Fig 2D). This suggested that GtmA activity may influence the production of other SM through the production of BmGT or, alternatively, by attenuating gli-cluster activity.

In order to uncover if the absence of BmGT was directly responsible for the metabolite alterations, the extended culture experiment was repeated and exogenous BmGT was added $(10 \mu \mathrm{g} /$ $\mathrm{ml}$ final) to A. fumigatus $\Delta$ gtmA prior to static incubation for 25 $\mathrm{d}(n=4)$. Methanol was added to the control cultures. Exogenously added BmGT did not result in the restoration of pseurotin A or fumagillin production in A. fumigatus $\Delta g t m A$ to wild-type levels, suggesting that BmGT does not directly facilitate the production of these metabolites (Fig 2E).

\subsection{Label-Free Quantitative (LFQ) proteomics of late-stage cultures} reveals that the absence of gtmA expression and resulting dysregulated gliotoxin biosynthesis leads to widespread proteomic alterations

The metabolomic analysis demonstrated that $A$. fumigatus $\triangle g t m A$ has an altered SM profile in comparison with that of the wild-type or gtm $A^{C}$ cultured under identical conditions. Comparative label-free quantitative (LFQ) proteomic analysis was carried out to further elucidate the involvement of GtmA activity in this metabolite profile alteration. A total of 1468 proteins were detected in A. fumigatus wild-type vs. $\Delta g t m A$ analysis (Fig 3). Two proteins were uniquely detected in A. fumigatus $\triangle \mathrm{gtmA}$ and 87 proteins were significantly more abundant in this mutant (Table 1). Proteins

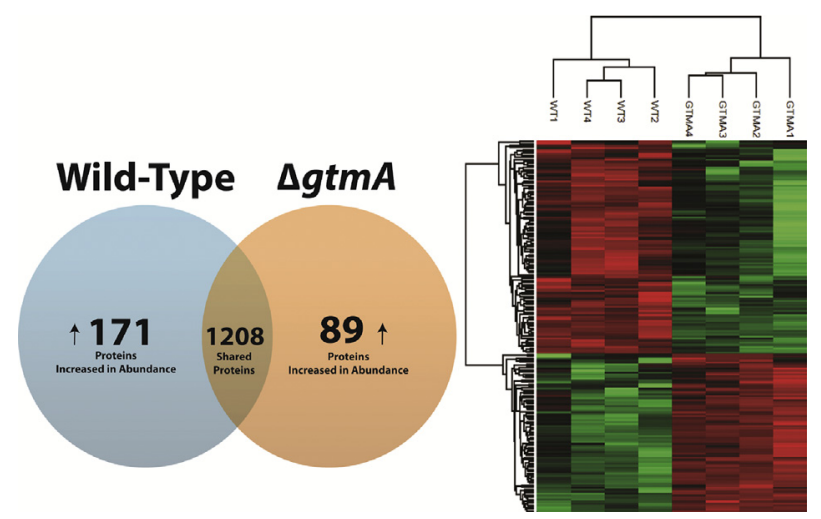

Fig 3. Venn-diagram illustrating the proteins with altered abundance in A. fumigatus wild-type when compared to $\Delta$ gtmA. Heat map depicting hierarchal clustered expression data of the 260 proteins of differential abundance in $\Delta g t m A$ compared to the wild-type.
( $n=35$ ) were not detected in A. fumigatus $\Delta g t m A$ and 136 proteins were significantly less abundant in this strain compared to wildtype (Table 2). This dataset was cross referenced with the $\operatorname{gtm} A^{C}$ vs. $\triangle g t m A$ dataset in order to confirm which of these changes were directly due to the absence of $g \operatorname{tm} A$. Based on this enrichment, 27 proteins were not detected in A. fumigatus $\Delta g t m A$ and 93 proteins were significantly less abundant in this strain. Two proteins were uniquely detected in A. fumigatus $\triangle \mathrm{gtmA}$ under this condition and 71 proteins were significantly more abundant in this mutant (Table S1 and S2).

Four proteins encoded by the gli cluster were shown to be significantly more abundant ( $\log _{2}$-fold) in $\Delta g t m A$ compared to the wild-type. The MFS gliotoxin efflux transporter GliA (AFUA_6G09710; 1.82652), the membrane dipeptidase GliJ (AFUA_6G09650; 1.66652), a predicted O-methyltransferase GliM (AFUA_6G09680; 1.62724) and the glutathione S-transferase GliG (AFUA_6G09690; 1.40425) were increased in abundance in $\Delta$ gtmA (Table S1). Additionally, a putative short chain dehydrogenase (AFUA_4G08710; 1.37478) and a ThiJ/PfpI family protein (AFUA_5G01430; 1.93416), which were previously shown to be induced by gliotoxin exposure were more abundant in this condition. RmtA (AFUA_1G06190; 1.06), a putative arginine methyltransferase previously shown to act as a global regulator in Aspergillus flavus, mediating broad effects on secondary metabolism and development in this organism (Satterlee et al., 2016), also exhibited significantly elevated abundance. However, its role in A. fumigatus has yet to be elucidated.

Co-incident with the reduced levels of selected SMs, several proteins for which cognate transcripts have previously been shown to be induced by hypoxia, or by exposure to neutrophils, were increased in abundance in A. fumigatus $\Delta g t m A$. Two proteins which are repressed by gliotoxin exposure and two which are repressed by hypoxia (Vödisch et al., 2011) were significantly decreased in abundance in $\Delta g t m A$. This may be a response to the sustained gliotoxin production in A. fumigatus $\Delta g \operatorname{tm} A$ as the abundance of these proteins is returned to wild-type levels in the complemented strain. Proteins which had been shown previously to be induced by hypoxia exposure were significantly more abundant in A. fumigatus $\Delta$ gtmA compared to the wild-type (Vödisch et al., 2011). These include a putative transaldolase (AFUA_5G09230; 1.58072), a putative glyceraldehyde 3-phosphate dehydrogenase (AFUA_5G01030; 1.34405), an argininosuccinate lyase (AFUA_3G07790; 1.34137), a 6phosphogluconate dehydrogenase (AFUA_6G08050; 1.31184), a putative mevalonate kinase (AFUA_4G07780; 1.07826), and an essential 1, 3-beta-glucanosyltransferase (AFUA_2G05340; 1.0025). Additionally, glutathione synthase (AFUA_5G06610; 1.67077), which is an ortholog of the Saccharomyces cerevisiae Gsh2 glutathione biosynthetic protein, known to be induced by oxidative stress in this organism (Sugiyama et al., 2000), was more abundant. Additionally, proteins formerly shown to be induced by neutrophil exposure were also more abundant in $\Delta$ gtmA (Sugui et al., 2008).

Table 1

Top 10 proteins with increased abundance in A. fumigatus $\Delta g t m A$ compared to wild-type following extended culture. Data sorted by fold change, in descending order.

\begin{tabular}{|c|c|c|c|c|}
\hline Protein description & $\log _{2}$ (Fold increase) & Peptides & Seq coverage [\%] & Protein IDs \\
\hline Aldo-keto reductase. In uncharacterised secondary metabolite cluster. & 2.85624 & 9 & 36.2 & AFUA_2G01410 \\
\hline Tyrosyl-tRNA synthetase, cytoplasm, nucleus localization & 2.85558 & 10 & 34.5 & AFUA_5G10640 \\
\hline $\mathrm{ABC}$ transporter; $\mathrm{Cdr} 1 \mathrm{~B}$, mutation causes increased azole sensitivity & 2.4345 & 18 & 16.2 & AFUA_1G14330 \\
\hline MFS monocarboxylate transporter, putative & 2.29482 & 2 & 4.5 & AFUA_3G03320 \\
\hline ABC multidrug transporter Mdr1 & 2.19999 & 25 & 25 & AFUA_5G06070 \\
\hline Aminotransferase family protein, putative & 2.09468 & 12 & 38 & AFUA_2G13295 \\
\hline Glycerate dehydrogenase & 2.01153 & 7 & 28 & AFUA_1G13630 \\
\hline ThiJ/PfpI family protein; abundant in conidia & 1.93416 & 9 & 55.1 & AFUA_5G01430 \\
\hline Glutamyl-tRNA(Gln) amidotransferase, subunit A & 1.93045 & 12 & 34.5 & AFUB_092380 \\
\hline MFS gliotoxin efflux transporter GliA, encoded in the gliotoxin biosynthetic gene cluster & 1.82652 & 4 & 9.2 & AFUA_6G09710 \\
\hline
\end{tabular}


Table 2

Top 10 proteins with decreased abundance in A. fumigatus $\Delta$ gtmA compared to wild-type following extended culture. Data sorted by fold change, in descending order.

\begin{tabular}{|c|c|c|c|c|}
\hline Protein description & $\begin{array}{l}\log _{2} \text { (Fold } \\
\text { decrease) }\end{array}$ & Peptides & $\begin{array}{l}\text { Seq coverage } \\
{[\%]}\end{array}$ & Protein IDs \\
\hline $\begin{array}{l}\text { Non-heme } \mathrm{Fe}(\mathrm{II}) \text { and alpha-ketoglutarate-dependent dioxygenase; catalyses the conversion of fumitremorgin } \\
\mathrm{B} \text { to verruculogen }\end{array}$ & -4.32466 & 21 & 87.3 & AFUA_8G00230 \\
\hline Phytanoyl-CoA dioxygenase family protein & -4.17772 & 12 & 46.8 & AFUA_8G00480 \\
\hline O-methyltransferase, putative & -3.85167 & 11 & 70.6 & AFUA_8G00390 \\
\hline IgE-binding protein & -3.76683 & 3 & 34.5 & AFUA_6G00430 \\
\hline $\begin{array}{l}\text { Putative methyltransferase; member of the pseurotin A gene cluster; conidia-enriched protein; hypoxia } \\
\text { induced protein }\end{array}$ & -3.28917 & 28 & 79.7 & AFUA_8G00550 \\
\hline Cysteine-rich secreted protein & -3.27599 & 15 & 63.3 & AFUA_7G01060 \\
\hline Conserved hypothetical protein, hypoxia induced protein & -3.24847 & 12 & 84.7 & AFUA_7G01060 \\
\hline DltD N-terminal domain protein (BF Unique) & -3.16375 & 15 & 78.4 & AFUA_8G00380 \\
\hline Glutathione S-transferase, putative & -2.96915 & 11 & 41 & AFUA_4G14380 \\
\hline Putative secreted 1,4-beta-D-glucan glucanhydrolase & -2.90284 & 27 & 53.3 & AFUA_7G06140 \\
\hline
\end{tabular}

These included a putative carbon-nitrogen family hydrolase (AFUA_5G02350; 1.567), a putative myo-inositol-phosphate synthase (AFUA_2G01010; 1.21826) and an aldehyde reductase (Akr1) (AFUA_6G10260; 1.09358). Mannitol 2-dehydrogenase which has a predicted role in mannitol metabolism (AFUA_4G14450; 1.52766) was also more abundant. Mannitol is an important virulence determinant of pathogenic fungi. Its high antioxidant capacity aids in suppressing the reactive oxygen species mediated attacks from neutrophils (Wyatt et al., 2014). This suggests that the sustained expression of the gli-cluster in long-term cultures may be translated as an oxidative challenge to A. fumigatus.

Six transporter proteins were found to be significantly more abundant in $\triangle$ gtmA. These were an $\mathrm{ABC}$ transporter Cdr1B (AFUA_1G14330; 2.4345), a putative MFS monocarboxylate transporter (AFUA_3G03320; 2.29482) (located in an uncharacterised SM cluster (Lind et al., 2016)), the ABC multidrug transporter Mdr1 (AFUA_5G06070; 2.19999), a putative plasma membrane $\mathrm{H}^{+}$ ATPase Pma1 (AFUA_3G07640; 1.3888), the low affinity plasma membrane zinc transporter ZrfB (AFUA_2G03860; 1.30673), which is induced by zinc depletion and the amino acid permease Gap1 (AFUA_7G04290; 1.0342). Pma1 was upregulated during conidial germination and in response to amphotericin $\mathrm{B}$ and downregulated by caspofungin treatment (Gautam et al., 2008; Cagas et al., 2011). Overexpression of the Cdr1B transporter was reported to be responsible for azole resistance in a clinical setting (Fraczek et al., 2013). The $\mathrm{Zn}_{2}-\mathrm{Cys}_{6}$ transcription factor AtrR was recently shown to be responsible for regulating cdr1B expression in A. fumigatus (Hagiwara et al., 2017).

Aminoacyl-tRNA synthetases are central enzymes in translation which provide the charged tRNAs needed for protein synthesis (Guo et al., 2010). Tyrosyl-tRNA synthetase (AFUA_5G10640; 2.85558), Seryl-tRNA synthetase (AFUA_5G05490; 1.22428), Putative valyltRNA synthetase (AFUA_8G04800; 1.22314), Isoleucyl-tRNA synthetase (AFUA_1G13710; 1.10632) and a putative Lysyl-tRNA synthetase (AFUA_6G07640; 1.05645) were significantly more abundant in A. fumigatus $\Delta g t m A$ compared to the wild-type. Several cell wallrelated proteins were increased in abundance in $A$. fumigatus $\Delta g t m A$. These included the GPI-anchored cell wall beta-1,3endoglucanase Bgt2 (AFUA_3G00270; 1.35218) and three $\beta(1-3$ ) glucanosyltransferases which belong to the 7-member GEL family (Fontaine et al., 2003; Gastebois et al., 2010); Gel5 (AFUA_8G02130; 1.23988), Gel3 (AFUA_2G12850; 1.00391), and Gel4 (AFUA_2G05340; 1.0025; previously shown to be increased in hypoxia). GlfA, a UDP-galactopyranose mutase (AFUA_3G12690; 1.3312 ), was also significantly more abundant. A. fumigatus $\Delta g l f A$ is devoid of galactofuranose and displays attenuated virulence in a lowdose mouse model of invasive aspergillosis (Schmalhorst et al., 2008). Interestingly, the small monomeric GTPase RasA (AFUA_5G11230; 1.65165) was also significantly more abundant in
$\Delta g t m A$. A $\Delta$ rasA mutant demonstrated a phenotype of cell wall instability and slow germination (Fortwendel et al., 2008). Higher levels of RasA abundance may be linked to the cell wall remodelling described above.

As mentioned above, A. fumigatus $\Delta g t m A 28 \mathrm{~d}$ cultures showed a significant decrease in the production of pseurotin $A$ and fumagillin compared to the wild-type strain. Complementary LFQ proteomic analysis revealed a significant decrease in abundance of several proteins encoded by the chromosome 8 supercluster (AFUA_8G00100-00720)(Wiemann et al., 2013), in agreement with the metabolomic analysis which revealed the decreased production of pseurotin A and fumagillin (Figs 2 and 4). A total of 15 proteins from this cluster were detected as less abundant in $\Delta g t m A$. Interestingly, 19 of the 98 proteins detected as significantly decreased in abundance in $\triangle g t m A$ are encoded on Chromosome 8, whereas 2 of the 38 proteins which were detected as increased in abundance in $\Delta g t m A$ were similarly located. O'Keeffe et al. (2014) demonstrated that an intact gliotoxin self-protection mechanism, mediated by GliT, is essential to regulate the biosynthesis of apparently unrelated metabolites such as pseurotin A, fumagillin and fumitremorgins. Herein, we have shown that when A. fumigatus cultures are incubated for extended duration, the absence of GtmA, resulting in dysregulated gliotoxin production, also has downstream effects on the biosynthesis of apparently unrelated natural products produced by this organism. A total of 17 proteins from the supercluster (Wiemann et al., 2013) were detected as less abundant in $\Delta$ gtmA. Interestingly, 23 of the 136 proteins detected as significantly decreased in abundance in $\triangle$ gtmA are encoded on Chromosome 8 . This is in comparison to 2 of the 72 proteins detected as increased in abundance in $\Delta g t m A$ being encoded on Chromosome 8. Several proteins encoded by the supercluster were either undetectable or significantly decreased in abundance in A. fumigatus $\Delta$ gtmA (Table 3; Table S2). Four proteins involved in the synthesis of fumitremorgins were significantly decreased in abundance in $\Delta$ gtmA. FtmPT1 (AFUA_8G00210; not detected) and FtmPT2 (AFUA_8G00250; not detected), two prenyltransferases involved in fumitremorgin B biosynthesis, FtmD, an $O$-methyltransferase involved in fumitremorgin B synthesis (AFUA_8G00200; not detected), and FtmF, an alpha-ketoglutarate-dependent dioxygenase which catalyses the conversion of fumitremorgin $B$ to verruculogen (AFUA_8G00230; -4.32466). Six proteins associated with fumagillin biosynthesis were not detected or significantly less abundant. These were the fumagillin polyketide synthase (FmaPKS) (AFUA_8G00370; not detected), the fumagillin phytanoylCoA-oxidase FmaF (AFUA_8G00480; -4.17772), FmaD, the fumagillin O-methyltransferase (AFUA_8G00390; -3.85167), a hypoxia induced protein; encoded in the fma gene cluster (AFUA_8G00430; -3.24847), FmaC, a putative fumagillin alpha/ beta hydrolase (AFUA_8G00380; -3.16375) and a putative 


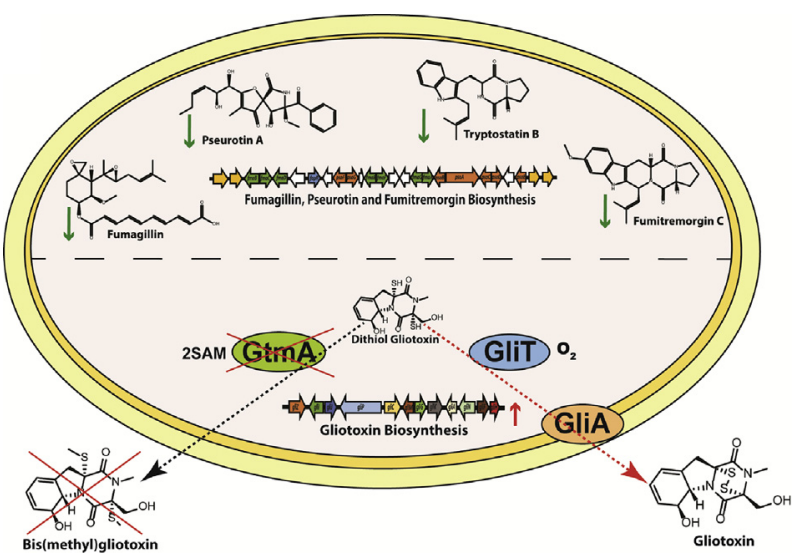

Fig 4. Absence of GtmA during long-term incubation of A. fumigatus results in the increased expression of the gli-cluster, widespread proteomic alterations and the decreased abundance of BGC-encoded enzymes located on chromosome 8. This in turn results in the reduced production of the respective compounds.

$O$-methyltransferase; encoded in the fma gene cluster (AFUA_8G00400; -2.85247). Four proteins involved in pseurotin A biosynthesis were significantly less abundant. PsoB, PsoD, PsoC, a putative pseurotin A methyltransferase (AFUA_8G0055 0 ; -3.28917), PsoA, the pseurotin A non-ribosomal peptide synthetase (AFUA_8G00540; - 1.60039), a pseurotin A dual-functional mono-oxygenase/methyltransferase PsoE (AFUA_8G00560; not detected) and PsoF, a putative pseurotin A dual methyltransferase/ monooxygenase (AFUA_8G00440; -2.74114) (Table 3; Table S2). A glutathione S-transferase (AFUA_4G14380; -2.96915) encoded within the helvolic acid cluster (AFUA_4G14380-4850) (Mitsuguchi et al., 2009) was also decreased in abundance in A. fumigatus $\Delta g t m A$.

Two proteins shown previously to be repressed by gliotoxin exposure (Carberry et al., 2012) were shown to be significantly decreased in abundance in $\triangle \operatorname{gtm} A$ : a mitochondrial peroxiredoxin (AFUA_4G08580; -1.96889) with a predicted role in cell redox homoeostasis regulation and a putative alcohol dehydrogenase
(AFUA_7G01000; -1.17862) involved in ethanol metabolism. A putative thioredoxin (AFUA_8G01090; not detected) and an M repeat protein (AFUA_6G08660; -1.99673), which were shown to be hypoxia-repressed were also decreased in expression (Vödisch et al., 2011) (Table S2). The thiol methyltransferase GtmA (Dolan et al., 2014, 2017) was uniquely detected in the wild-type condition and abundance was restored in the complemented strain. Several cell wall associated proteins were also not detected or decreased in abundance in A. fumigatus $\Delta$ gtmA mutant in comparison with the wild-type. These included the conidial hydrophobin RodA (AFUA_5G09580; not detected), a putative glycophosphatidylinositol (GPI)-anchored cell wall protein MP-2 (AFUA_2G05150; not detected) and a putative phiA family cell wall protein (AFUA_3G03060; -1.5461).

Several mitochondrial-associated proteins were not detected or less abundant in $\Delta g t m A$. These included an uncharacterized protein (AFUA_1G13195; not detected) with orthologs involved in cristae formation and integral to mitochondrial inner membrane, a putative iron-sulphur cluster biosynthesis protein extrinsic to mitochondrial inner membrane (AFUA_3G06492, not detected), a putative mitochondrial intermembrane space translocase subunit (AFUA_1G04470; 1.97737), a putative mitochondrial peroxiredoxin (AFUA_4G08580; -1.96889) with a predicted role in cell redox homoeostasis regulation, a putative prohibitin (AFUA_2G0 $9090 ;-1.5947)$ with orthologs involved in mitochondrion inheritance, a putative mitochondrial 2-oxodicarboxylate carrier protein (AFUA_1G09660; -1.56908), a putative adenylate kinase with mitochondrial intermembrane space localization (AFUA_1G07 $530 ;-1.4474)$, a putative outer mitochondrial membrane protein porin (AFUA_4G06910; -1.31711), a putative mitochondrial genome maintenance protein Mgm101 (AFUA_2G09560; 1.26767), a mitochondrial glycerol-3-phosphate dehydrogenase (AFUA_1G $08810 ;-1.19813$ ) and a putative mitochondrial processing peptidase alpha subunit with a role in protein processing involved in protein targeting to mitochondrion (AFUA_1G11870; -1.1768). Interestingly, several hypoxia-induced proteins were either not detected or decreased in abundance in A. fumigatus $\Delta \mathrm{gtmA}$ (Blatzer et al., 2011). These included a gamma-glutamyltranspeptidase (AFUA_4G13580, not detected); SrbA-regulated during hypoxia,

Table 3

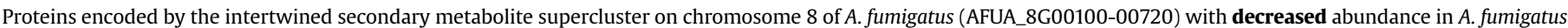
$\Delta g t m A$ compared to wild-type grown for $28 \mathrm{~d}$ in Czapek-Dox media. Data sorted by fold change, in descending order.

\begin{tabular}{|c|c|c|c|c|}
\hline Protein description & $\begin{array}{l}\log _{2} \text { (Fold } \\
\text { decrease) }\end{array}$ & Peptides & $\begin{array}{l}\text { Seq } \\
\text { coverage } \\
{[\%]}\end{array}$ & Protein IDs \\
\hline Putative prenyltransferase involved in fumitremorgin B biosynthesis & Absent & 16 & 39.8 & AFUA_8G00250 \\
\hline $\begin{array}{l}\text { Polyketide synthase (PKS), encoded in the fma (fumagillin) secondary metabolite gene cluster; required for } \\
\text { fumagillin biosynthesis }\end{array}$ & Absent & 27 & 15.4 & AFUA_8G00370 \\
\hline Protein of unknown function & Absent & 8 & 18.2 & AFUA_8G00630 \\
\hline translation elongation factor eEF-1, gamma subunit, putative & Absent & 9 & 36.7 & AFUA_8G00580 \\
\hline $\begin{array}{l}\text { Putative brevianamide F prenyltransferase, predicted to convert brevianamide F to tryprostatin B; involved in the } \\
\text { biosynthesis of fumitremorgins }\end{array}$ & Absent & 13 & 32.3 & AFUA_8G00210 \\
\hline Putative O-methyltransferase with a predicted role in fumitremorgin B synthesis & Absent & 22 & 68.9 & AFUA_8G00200 \\
\hline $\begin{array}{l}\text { Non-heme } \mathrm{Fe}(\mathrm{II}) \text { and alpha-ketoglutarate-dependent dioxygenase; catalyses the conversion of fumitremorgin } \mathrm{B} \text { to } \\
\text { verruculogen }\end{array}$ & -4.32466 & 21 & 87.3 & AFUA_8G00230 \\
\hline Putative iron-dependent oxygenase; encoded in the fma (fumagillin) secondary metabolite gene cluster & -4.17772 & 12 & 46.8 & AFUA_8G00480 \\
\hline Predicted O-methyltransferase; encoded in the fma (fumagillin) secondary metabolite gene cluster & -3.85167 & 11 & 70.6 & AFUA_8G00390 \\
\hline $\begin{array}{l}\text { Putative methyltransferase; member of the pseurotin A gene cluster; conidia-enriched protein; hypoxia induced } \\
\text { protein }\end{array}$ & -3.28917 & 28 & 79.7 & AFUA_8G00550 \\
\hline Hypoxia induced protein; encoded in the fma (fumagillin) secondary metabolite gene cluster & -3.24847 & 12 & 84.7 & AFUA_8G00430 \\
\hline Putative alpha/beta hydrolase; encoded in the fma (fumagillin) secondary metabolite gene cluster & -3.16375 & 15 & 78.4 & AFUA_8G00380 \\
\hline Protein of unknown function; encoded in the fma (fumagillin) secondary metabolite gene cluster & -2.85247 & 10 & 45.1 & AFUA_8G00400 \\
\hline $\begin{array}{l}\text { Baeyer-Villiger monooxygenase (BVMO); hypoxia induced protein; encoded in the fma (fumagillin) secondary } \\
\text { metabolite gene cluster }\end{array}$ & -2.74114 & 41 & 63.4 & AFUA_8G00440 \\
\hline $\begin{array}{l}\text { Non-ribosomal peptide synthetase (NRPS); PKS/NRPS hybrid; multidomain protein; required for pseurotin A } \\
\text { production; transcript induced by voriconazole; induced by hypoxia and in infected mouse lungs }\end{array}$ & -1.60039 & 105 & 36.9 & AFUB_086030 \\
\hline
\end{tabular}


Putative flavohemoprotein (AFUA_4G03410; -1.42304), Putative outer mitochondrial membrane protein porin (AFUA_4G06 $910 ;-1.31711)$, Ubiquinol-cytochrome c reductase iron-sulphur subunit precursor (AFUA_5G10610; -1.19224) with a predicted role in oxidative phosphorylation, an aspartic acid endopeptidase (AFUA_3G11400; -1.18445) and a thiamine biosynthesis protein (AFUA_5G02470; -1.11766).

Gliotoxin can act as a sporulation signal for Aspergillus nidulans development under mildly reducing conditions through NapA oxidative stress regulation, as reflected by green conidial pigmentation (Zheng et al., 2015). Although no phenotypic differences were noted for $\Delta g t m A$, it is conceivable that the absence of gliotoxin bis-thiomethylation can alter the normal course of fungal development by sustaining the gli-cluster activation signal. It is speculated that this may then lead to downstream redox stress signalling, cell wall remodelling and the modification of secondary metabolite profiles.

\section{Conclusions}

Overall these data show that the loss of GtmA-mediated thiomethylation upon extended culture duration has downstream effects on seemingly unrelated BGC-encoded metabolites in A. fumigatus. The exact mechanism of this effect is unclear, however, it is likely that the sustained gli-cluster activation in the absence of GtmA artificially prioritises the expression of this cluster, resulting in extensive downstream proteomic remodelling, which occurs at the expense of other BGCs in this organism. We also explored the possibility that BmGT itself acts as a signal to sustain the expression of the unrelated BGC-encoded metabolites, which decrease in its absence. Adding a high concentration $(10 \mu \mathrm{g} / \mathrm{mL})$ of BmGT to the cultures did not complement the phenotype, suggesting that BmGT itself does not sustain the expression of these unrelated clusters. It's important to note that although $A$. fumigatus exposure to gliotoxin results in a rapid intracellular accumulation due to the redox properties of this metabolite (Bernardo et al., 2003), BmGT does not have this capability due to the absence of the characteristic disulfide bridge. This means that we cannot completely rule out that the intracellular accumulation of BmGT is responsible this phenotype. However, as shown in our earlier study, exposure of $A$. fumigatus to $5 \mu \mathrm{g} / \mathrm{ml} \mathrm{BmGT}$ results in a distinct proteomic response, increasing the GliT and GtmA protein abundance 2 fold (Dolan et al., 2014). This would suggest that despite its inability to accumulate intracellularly to the same extent as gliotoxin, exogenously added BmGT can act as a signalling molecule in A. fumigatus when applied exogenously.

Although several master regulators have been shown to orchestrate secondary metabolism in fungi, this work highlights the importance of the BGC-encoded metabolites themselves as important signals in rewiring SM production. Despite our extensive functional and mechanistic insight into how these BGC-encoded metabolites are synthesised, the precise mechanism of how intracellular signals orchestrate temporal control over SM production have yet to be understood. Further work will focus on the precise nature of how exactly these competing metabolic signals are integrated, resulting in the expression of a 'typical' secondary metabolome of A. fumigatus and other pathogenic fungi.

\section{Acknowledgements}

This work was funded by a Science Foundation Ireland Principal Investigator Award to SD (PI/11/1188). SKD was a recipient of an Irish Research Council Embark PhD Fellowship. LC-MS facilities were funded by competitive awards from Science Foundation Ireland (12/RI/2346 (3)) and the Irish Higher Education Authority.

\section{Appendix A. Supplementary data}

Supplementary data related to this article can be found at https://doi.org/10.1016/j.funbio.2017.12.007.

\section{References}

Bernardo, P.H., Brasch, N., Chai, C.L.L., Waring, P., 2003. A novel redox mechanism for the glutathione-dependent reversible uptake of a fungal toxin in cells. J. Biol. Chem. 278, 46549-46555. https://doi.org/10.1074/jbc.M304825200.

Bignell, E., Cairns, T.C., Throckmorton, K., Nierman, W.C., Keller, N.P., 2016. Secondary metabolite arsenal of an opportunistic pathogenic fungus. Philos. Trans. R. Soc. Lond. B Biol. Sci. 371.

Blatzer, M., Barker, B.M., Willger, S.D., Beckmann, N., Blosser, S.J., Cornish, E.J. Mazurie, A., Grahl, N., Haas, H., Cramer, R.A., 2011. SREBP coordinates iron and ergosterol homeostasis to mediate triazole drug and hypoxia responses in the human fungal pathogen Aspergillus fumigatus. PLoS Genet. 7, e1002374 https:// doi.org/10.1371/journal.pgen.1002374.

Cagas, S.E., Jain, R., Li, H., Perlin, D.S., 2011. Profiling the Aspergillus fumigatus proteome in response to caspofungin. Antimicrob. Agents Chemother. 55, 146-154. https://doi.org/10.1128/AAC.00884-10.

Carberry, S., Molloy, E., Hammel, S., O'Keeffe, G., Jones, G.W., Kavanagh, K., Doyle, S., 2012. Gliotoxin effects on fungal growth: mechanisms and exploitation. Fungal Genet. Biol. 49, 302-312.

Choi, E.J., Park, J.-S., Kim, Y.-J., Jung, J.-H., Lee, J.K., Kwon, H.C., Yang, H.O., 2011. Apoptosis-inducing effect of diketopiperazine disulfides produced by Aspergillus sp. KMD 901 isolated from marine sediment on HCT116 colon cancer cel lines. J. Appl. Microbiol. 110, 304-313. https://doi.org/10.1111/j.13652672.2010.04885.x.

Cox, J., Mann, M., 2008. MaxQuant enables high peptide identification rates, individualized p.p.b.-range mass accuracies and proteome-wide protein quantification. Nat. Biotechnol. 26, 1367-1372. https://doi.org/10.1038/nbt.1511.

Dolan, S.K., Bock, T., Hering, V., Owens, R.A., Jones, G.W., Blankenfeldt, W., Doyle, S. 2017. Structural, mechanistic and functional insight into gliotoxin bis-thiomethylation in Aspergillus fumigatus. Open Biol. 7, 160292.

Dolan, S.K., O'Keeffe, G., Jones, G.W., Doyle, S., 2015. Resistance is not futile: Gliotoxin biosynthesis, functionality and utility. Trends Microbiol. 1-10. https:// doi.org/10.1016/j.tim.2015.02.005.

Dolan, S.K., Owens, R.A., O'Keeffe, G., Hammel, S., Fitzpatrick, D.A., Jones, G.W., Doyle, S., 2014. Regulation of nonribosomal peptide synthesis: bisthiomethylation attenuates gliotoxin biosynthesis in Aspergillus fumigatus. Chem. Biol. 21, 999-1012. https://doi.org/10.1016/j.chembiol.2014.07.006.

Fontaine, T., Magnin, T., Melhert, A., Lamont, D., Latge, J.P., Ferguson, M.A.J., 2003. Structures of the glycosylphosphatidylinositol membrane anchors from Aspergillus fumigatus membrane proteins. Glycobiology 13, 169-177. https://doi.org/ 10.1093/glycob/cwg004.

Forseth, R.R., Fox, E.M., Chung, D., Howlett, B.J., Keller, N.P., Schroeder, F.C., 2011 Identification of cryptic products of the gliotoxin gene cluster using NMR-based comparative metabolomics and a model for gliotoxin biosynthesis. J. Am. Chem. Soc. 133, 9678-9681. https://doi.org/10.1021/ja2029987.

Fortwendel, J.R., Fuller, K.K., Stephens, T.J., Bacon, W.C., Askew, D.S., Rhodes, J.C., 2008. Aspergillus fumigatus RasA regulates asexual development and cell wall integrity. Eukaryot. Cell 7, 1530-1539. https://doi.org/10.1128/EC.00080-08.

Fraczek, M.G., Bromley, M., Buied, A., Moore, C.B., Rajendran, R., Rautemaa, R., Ramage, G., Denning, D.W., Bowyer, P., 2013. The cdr1B efflux transporter is associated with non-cyp51a-mediated itraconazole resistance in Aspergillus fumigatus. J. Antimicrob. Chemother. 68, 1486-1496. https://doi.org/10.1093/ jac/dkt075.

Gastebois, A., Fontaine, T., Latgé, J.-P., Mouyna, I., 2010. beta(1-3)Glucanosyltransferase Gel4p is essential for Aspergillus fumigatus. Eukaryot. Cell 9, 1294-1298. https://doi.org/10.1128/EC.00107-10.

Gautam, P., Shankar, J., Madan, T., Sirdeshmukh, R., Sundaram, C.S., Gade, W.N., Basir, S.F., Sarma, P.U., 2008. Proteomic and transcriptomic analysis of Aspergillus fumigatus on exposure to amphotericin B. Antimicrob. Agents .Chemother. 52, 4220-4227. https://doi.org/10.1128/AAC.01431-07.

Guo, M., Yang, X.-L., Schimmel, P., 2010. New functions of aminoacyl-tRNA synthetases beyond translation. Nat. Rev. Mol. Cell Biol. 11, 668-674. https:/ doi.org/10.1038/nrm2956.

Guo, C.-J., Yeh, H.-H., Chiang, Y.-M., Sanchez, J.F., Chang, S.-L., Bruno, K.S. Wang, C.C.C., 2013. Biosynthetic pathway for the epipolythiodioxopiperazine acetylaranotin in Aspergillus terreus revealed by genome-based deletion analysis. J. Am. Chem. Soc. 135, 7205-7213. https://doi.org/10.1021/ja3123653.

Hagiwara, D., Miura, D., Shimizu, K., Paul, S., Ohba, A., Gonoi, T., Watanabe, A. Kamei, K., Shintani, T., Moye-Rowley, W.S., Kawamoto, S., Gomi, K., 2017. A Novel Zn 2-Cys 6 Transcription Factor AtrR Plays a Key Role in an Azole Resistance Mechanism of Aspergillus fumigatus by Co-regulating cyp51A and cdr1B Expressions. https://doi.org/10.1371/journal.ppat.1006096.

Haritakun, R., Rachtawee, P., Komwijit, S., Nithithanasilp, S., Isaka, M., 2012. Highly conjugated ergostane-type steroids and aranotin-type diketopiperazines from the fungus Aspergillus terreus BCC 4651. Helv. Chim. Acta 95, 308-313. https:// doi.org/10.1002/hlca.201100335.

Hou, L., Mori, D., Takase, Y., Meihua, P., Kai, K., Tokunaga, O., 2009. Fumagillin inhibits colorectal cancer growth and metastasis in mice: in vivo and in vitro study 
of anti-angiogenesis. Pathol. Int. 59, 448-461. https://doi.org/10.1111/j.14401827.2009.02393.x.

Komagata, D., Fujita, S., Yamashita, N., Saito, S., Morino, T., 1996. Novel neuritogenic activities of pseurotin A and penicillic acid. J. Antibiot. 49, 958-959.

Liang, W.L., Le, X., Li, H.J., Yang, X.L., Chen, J.X., Xu, J., Liu, H.L., Wang, L.Y., Wang, K.T. Hu, K.C., Yang, D.P., Lan, W.J., 2014. Exploring the chemodiversity and biological activities of the secondary metabolites from the marine fungus Neosartorya pseudofischeri. Marine Drugs 12, 5657-5676. https://doi.org/10.3390/ md12115657.

Li, Y., Yue, Q., Krausert, N.M., An, Z., Gloer, J.B., Bills, G.F., 2016. Emestrins: anticryptococcus epipolythiodioxopiperazines from Podospora australis. J. Nat. Prod. 79, 2357-2363. https://doi.org/10.1021/acs.jnatprod.6b00498.

Lind, A.L., Smith, T.D., Saterlee, T., Calvo, A.M., Rokas, A., 2016. Regulation of sec ondary metabolism by the velvet complex is temperature-responsive in Aspergillus. G3 (Bethesda, Md.) 6, 4023-4033. https://doi.org/10.1534/ g3.116.033084.

Lind, A.L., Wisecaver, J.H., Lameiras, C., Wiemann, P., Palmer, J.M., Keller, N.P., Rodrigues, F., Goldman, G.H., Rokas, A., 2017. Drivers of genetic diversity in secondary metabolic gene clusters within a fungal species. PLoS Biol. 15, e2003583.

Maebayashi, Y., Horie, Y., Satoh, Y., Yamazaki, M., 1985. Isolation of pseurotin A and a new pyrazine from Pseudallescheria boydii. Mycotoxins 1985, 33-34. https:// doi.org/10.2520/myco1975.1985.22 33.

Mitsuguchi, H., Seshime, Y., Fujii, I., Shibuya, M., Ebizuka, Y., Kushiro, T., 2009. Biosynthesis of steroidal antibiotic fusidanes: functional analysis of oxidosqualene cyclase and subsequent tailoring enzymes from Aspergillus fumigatus. J. Am. Chem. Soc. 131, 6402-6411.

Nützmann, H.-W., Reyes-Dominguez, Y., Scherlach, K., Schroeckh, V., Horn, F., Gacek, A., Schümann, J., Hertweck, C., Strauss, J., Brakhage, A.A., 2011. Bacteriainduced natural product formation in the fungus Aspergillus nidulans requires Saga/Ada-mediated histone acetylation. Proc. Natl. Acad. Sci. U.S.A. 108, 14282-14287. https://doi.org/10.1073/pnas.1103523108.

O'Keeffe, G., Hammel, S., Owens, R.A., Keane, T.M., Fitzpatrick, D.A., Jones, G.W. Doyle, S., 2014. RNA-seq reveals the pan-transcriptomic impact of attenuating the gliotoxin self-protection mechanism in Aspergillus fumigatus. BMC Genom. 15 (894) https://doi.org/10.1186/1471-2164-15-894.

Ochi, K., Hosaka, T., 2013. New strategies for drug discovery: activation of silent or weakly expressed microbial gene clusters. Appl. Microbiol. Biotechnol. 97, 87-98. https://doi.org/10.1007/s00253-012-4551-9.

Perrin, R.M., Fedorova, N.D., Jin, W.B., Cramer, R.A., Wortman, J.R., Kim, H.S. Nierman, W.C., Keller, N.P., 2007. Transcriptional regulation of chemical diversity in Aspergillus fumigatus by LaeA. PLoS Pathog. 3, 508-517. https:// doi.org/10.1371/journal.ppat.0030050.

Satterlee, T., Cary, J.W., Calvo, A.M., 2016. RmtA, a putative arginine methyltransferase, regulates secondary metabolism and development in Aspergillus flavus (GH Goldman, Ed.). PLOS ONE 11, e0155575. https://doi.org/10.1371/ journal.pone.0155575.

Scharf, D.H., Habel, A., Heinekamp, T., Brakhage, A.A., Hertweck, C., 2014. Opposed effects of enzymatic gliotoxin N- and S-methylations. J. Am. Chem. Soc. 136, 11674-11679. https://doi.org/10.1021/ja5033106.

Schmalhorst, P.S., Krappmann, S., Vervecken, W., Rohde, M., Müller, M., Braus, G.H., Contreras, R., Braun, A., Bakker, H., Routier, F.H., 2008. Contribution of galactofuranose to the virulence of the opportunistic pathogen Aspergillus fumigatus. Eukaryot. Cell 7, 1268-1277. https://doi.org/10.1128/EC.00109-08.

Schueffler, A., Anke, T., 2014. Fungal natural products in research and development. Nat. Prod. Rep. 1425-1448. https://doi.org/10.1039/C4NP00060A.

Sin, N., Meng, L., Wang, M.Q., Wen, J.J., Bornmann, W.G., Crews, C.M., 1997. The antiangiogenic agent fumagillin covalently binds and inhibits the methionine aminopeptidase, MetAP-2. Proc. Natl. Acad. Sci. U.S.A. 94, 6099-6103.

Sugiyama, K., Izawa, S., Inoue, Y., 2000. The Yap1p-dependent induction of glutathione synthesis in heat shock response of Saccharomyces cerevisiae. J. Biol. Chem. 275, 15535-15540.

Sugui, J.A., Kim, H.S., Zarember, K.A., Chang, Y.C., Gallin, J.I., Nierman, W.C., KwonChung, K.J., 2008. Genes differentially expressed in conidia and hyphae of Aspergillus fumigatus upon exposure to human neutrophils. PLos One 3, e2655. https://doi.org/10.1371/journal.pone.0002655.

Suzuki, Y., Takahashi, H., Esumi, Y., Arie, T., Morita, T., Koshino, H., Uzawa, J., Uramoto, M., Yamaguchi, I., 2000. Haematocin, a new antifungal diketopiperazine produced by Nectria haematococca Berk. et Br. (880701a-1) causing nectria blight disease on ornamental plants. J. Antibiot. 53, 45-49.

Vödisch, M., Scherlach, K., Winkler, R., Hertweck, C., Braun, H.-P., Roth, M., Haas, H., Werner, E.R., Brakhage, A.A., Kniemeyer, O., 2011. Analysis of the Aspergillus fumigatus proteome reveals metabolic changes and the activation of the pseurotin a biosynthesis gene cluster in response to hypoxia. J. Proteome Res. 10, 2508-2524. https://doi.org/10.1021/pr1012812.

Wenke, J., Anke, H., Sterner, O., 1993. Pseurotin A and 8-O-Demethylpseurotin A from Aspergillus fumigatus and their inhibitory activities on chitin synthase. Biosc. Biotech. Biochem. 57, 961-964. https://doi.org/10.1271/bbb.57.961.

Wiemann, P., Guo, C.-J., Palmer, J.M., Sekonyela, R., Wang, C.C.C., Keller, N.P., 2013. Prototype of an intertwined secondary-metabolite supercluster. Proc. Natl. Acad. Sci. U.S.A. 110, 17065-17070. https://doi.org/10.1073/pnas.1313258110.

Wyatt, T.T., van Leeuwen, M.R., Wösten, H.A.B., Dijksterhuis, J., 2014. Mannitol is essential for the development of stress-resistant ascospores in Neosartorya fischeri (Aspergillus fischeri). Fungal Genet. Biol. 64, 11-24. https://doi.org/ 10.1016/j.fgb.2013.12.010.

Zheng, H., Kim, J., Liew, M., Yan, J.K., Herrera, O., Bok, J.W., Kelleher, N.L., Keller, N.P. Wang, Y., 2015. Redox metabolites signal polymicrobial biofilm development via the NapA oxidative stress cascade in Aspergillus. Curr. Biol. 25, 29-37. https://doi.org/10.1016/j.cub.2014.11.018. 